\title{
Introduction to the special issue on Advances in Manufacturing Systems
}

\author{
T. van Woensel • J. Buzacott • S. Gershwin • \\ G. Liberopoulos · J. MacGregor Smith · S. Meerkov • \\ B. Tan
}

Published online: 19 May 2010

(C) The Author(s) 2010. This article is published with open access at Springerlink.com

The origin of this volume of the AOR stems from the Sixth International Conference on "Analysis of Manufacturing Systems" in Lunteren, The Netherlands, May 11-16, 2007. The Conference was jointly organized under the auspices of the Technische Universiteit Eindhoven (TU/e), the BETA Research School for Operations Management and Logistics, and EURANDOM, and was sponsored by BETA, EURANDOM, KNAW, and NWO. The head of the organizing committee was Dr. Tom Van Woensel of TU/e. The previous five

\footnotetext{
T. van Woensel ( $₫)$

Eindhoven University of Technology, Eindhoven, The Netherlands

e-mail: t.v.woensel@tue.nl

J. Buzacott

York University, Toronto, Canada

e-mail: jbuzacot@schulich.yorku.ca

S. Gershwin

Massachusetts Institute of Technology, Cambridge, USA

e-mail: gershwin@mit.edu

G. Liberopoulos

University of Thessaly, Thessaly, Greece

e-mail: glib@mie.uth.gr

J. MacGregor Smith

University of Massachusetts at Amherst, Amherst, USA

e-mail: jmsmith@ecs.umass.edu

S. Meerkov

University of Michigan, Ann Arbor, USA

e-mail: smm@eecs.umich.edu

B. Tan

Koç University, Istanbul, Turkey

e-mail: btan@ku.edu.tr
} 
biannual conferences were successfully organized on different Greek islands by Professor Chrissoleon Papadopoulos of the Aristotle University of Thessaloniki, Greece, so this was the first time that the conference was held outside Greece. The primary orientation of this series of conferences is the reporting of new research in the general area of mathematical modeling, analysis, design, and optimization of manufacturing systems. The scope of the conference is to serve as a forum for researchers, academics, and industrialists in the broad area of manufacturing systems analysis to discuss their most recent research findings and to provide them with an opportunity for technology transfer. The conference attracted fortyone international participants that presented their work. Professor Yves Dallery of Ecole Central Paris, France, gave the plenary talk.

The aim of this Volume is to accomplish a wider dissemination of the results reported in the conference, along with those obtained by other researchers in the field, for the benefit of the community of both theoreticians and practitioners. The volume consists of nine papers that deal with specific topics in the analysis of manufacturing systems. All papers were reviewed in accordance to the standard, rigorous AOR procedures.

The paper by Tan and Gershwin considers a Markovian fluid flow system with two stages separated by a finite buffer. By using the methodology they developed to analyze general Markovian continuous flow systems with a finite buffer, they model and analyze a range of models studied in the literature including systems with phase-type failure and repair-time distributions, systems with machines that have multiple up and down states, and systems with multiple unreliable machines in series or parallel in each stage.

Colledani and Tolio propose a decomposition method for evaluating the performance of transfer lines where machines can fail in multiple modes and can be repaired with nonexponential times. Indeed, while times to machine failure often can be modeled using exponential distributions with acceptable accuracy, times to repair are very rarely observed to be exponentially distributed in actual systems. The discrete acyclic phase-type distribution is used to model the repair process, for each failure mode of the machines composing the system. Numerical results show the high accuracy of the developed method in estimating the average throughput and buffer levels.

Many uncertain factors, such as machine breakdowns/repairs and customer demand, inherent in manufacturing systems make it difficult for managers to plan and control production processes. The paper by Sethi, Khmelnitsky, and Presman uses variational analysis to develop the necessary conditions of optimality and uses these to show the optimality of threshold-type policies for important classes of manufacturing systems.

Kat and Avsar study dynamic scheduling of multi-class systems by deriving the equivalence relations between the cost model to minimize the expected average inventory holding and backorder costs and the service model to minimize expected average inventory holding cost under an aggregate fill rate constraint. Based on the investigation that the optimal policy for the cost model is a base-stock policy with switching curves, they propose accurate heuristics by using an alternative service model to minimize basestock investment, instead of average inventory holding cost, under an aggregate fill rate constraint.

Meerkov and Zhang analyze serial production lines with machines having their quality characteristics monotonically decreasing as a function of their efficiency. They show that in such systems the production rate of good parts is a non-monotonic function with respect to machine efficiencies. Thus, the bottlenecks of these systems can be eliminated by either increasing or decreasing machine efficiency, depending on the operating point.

An approximation is proposed by Topan and Avsar to evaluate the steady-state performance of kanban controlled two-stage assembly systems. The proposed approximate, aggregate Markov chain formulation is decomposed into submodels, each with a product-form 
steady-state distribution. The submodels are then combined in such a way that the size of the problem becomes independent of the number of kanbans, leading to the computational advantage in using numerical matrix-geometric solution algorithms. Numerical analysis shows that the proposed approximation performs well in terms of accuracy and computational burden.

Tancrez, Chevalier and Semal examine flow lines with finite capacity and with general service time distributions. They propose and study a new approach, called "probability masses fitting" (PMF), in the preliminary step of the analysis, to build tractable discrete phase-type distributions. Several interesting properties are shown. The PMF is bounding, refinable, conserves the shape of the distributions, and leads to accurate performance evaluation.

The paper by Helber, Schimmelpfeng, Stolletz, and Lagershausen presents a linear programming approach to simultaneously evaluate and optimize flow lines with limited buffer capacity. They convert simulated processing times into sampled per-period production capacities of a large production-inventory model in discrete time. Thus simulation is embedded into a linear program. The approach is surprisingly precise unless buffers are very small or processing times are highly variable.

A decomposition/aggregation method is proposed by Mhada and Malhamé for the performance analysis of single part unreliable transfer lines under CONWIP disciplines. For a CONWIP loop with $(n-1)$ machines, the approximate model consists of $(n-1)$ building blocks each representing the complete CONWIP loop as seen from one of its $(n-1)$ distinct internal buffers. There is also one such building block associated with the total work-inprocess in the loop and used to evaluate the loop performance. The accuracy of performance estimates is validated via Monte Carlo simulations.

In closing, we wish to thank the numerous anonymous referees who gave of their valuable time to review the papers in this volume. Finally, we wish to thank Professor Endre Boros, Editor-in-Chief of AOR, and Katie D'Agosta, Managing Editor of AOR, for supporting the volume and offering their expert guidance and efforts to make this volume of the Annals a published reality. 